\title{
Broad characterization of endogenous peptides in the tree shrew visual system
}

\author{
Vaclav Ranc ${ }^{a, b}$, Filomena Petruzziello ${ }^{a}$, Robert Kretz ${ }^{a}$, Enrike G. Argandoña ${ }^{c}$, \\ Xiaozhe Zhang ${ }^{a, *}$, Gregor Rainer ${ }^{a}$ \\ ${ }^{a}$ University of Fribourg, Department of Medicine, Fribourg, Switzerland \\ ${ }^{b}$ Palacky University Olomouc, Department of Analytical Chemistry, Olomouc, Czech Republic \\ 'Unit of Anatomy, Department of medicine, University of Fribourg, Fribourg, Switzerland
}

\begin{abstract}
Endogenous neuropeptides, acting as neurotransmitters or hormones in the brain, carry out important functions including neural plasticity, metabolism and angiogenesis. Previous neuropeptide studies have focused on peptide-rich brain regions such as the striatum or hypothalamus. Here we present an investigation of peptides in the visual system, composed of brain regions that are generally less rich in peptides, with the aim of providing the first broad overview of peptides involved in mammalian visual functions. We target three important parts of the visual system: the primary visual cortex (V1), lateral geniculate nucleus (LGN) and superior colliculus (SC). Our study is performed in the tree shrew, a close relative of primates. Using a combination of data dependent acquisition and targeted LC-MS/MS based neuropeptidomics; we identified a total of 52 peptides from the tree shrew visual system. A total of 26 peptides, for example GAV and neuropeptide $\mathrm{K}$ were identified in the visual system for the first time. Out of the total 52 peptides, 27 peptides with high signal-to-noise-ratio $(>10)$ in extracted ion chromatograms (EIC) were subjected to label-free quantitation. We observed generally lower abundance of peptides in the LGN compared to V1 and SC. Consistently, a number of individual peptides showed high abundance in V1 (such as neuropeptide Y or somatostatin 28) and in SC (such as somatostatin 28 AA1-12). This study provides the first in-depth characterization of peptides in the mammalian visual system. These findings now permit the investigation of neuropeptide-regulated mechanisms of visual perception.
\end{abstract}

\section{Introduction}

Neuropeptides are evolutionarily old neurotransmitters present in all species possessing a nervous system [1]. They are involved in regulating many physiological pathways [2-6]. Their functions and expression levels can differ considerably across particular brain areas [7]. In the visual system, peptides were found to carry out important functions related to development [8], neural plasticity, regulation of blood flow and energy metabolism $[7,9]$. Neuropeptides also play a key role in the development and function of the inhibitory circuits that shape the visual system in response to experience [10] and their expression patterns are different in relation to morphologically and physiologically distinct interneuron classes [11].

\footnotetext{
* Corresponding author at: Visual Cognition Laboratory, Department of Medicine, University of Fribourg, Chemin de Musee 5, Fribourg, CH-1700, Switzerland. Tel.: +41 263008910; fax: +41 263009734.

E-mail address: xiaozhe.zhang@unifr.ch (X. Zhang).
} 
To advance our understanding of peptide functions in the visual system, we sought to provide an in-depth simultaneous characterization of a large set of peptides present in different substructures of the visual system. Compared to peptides previously identified in peptide-rich areas such as hypothalamus [12] or striatum [13], peptides in the visual system have so far received relatively little attention using mass spectrometry approaches.

Neuropeptides, from an analytical chemistry point of view, have been characterized predominantly by employing immunoassay or radioimmunoassay $[14,15]$. Classical immunochemical approaches are usually used to characterize only a few peptides or peptide families within a single study, but it is usually difficult to distinguish between similar peptides from one peptide family. Contrastingly to mass spectrometric approaches, immunohistochemical or radiography also cannot provide a complete image of peptides in the studied brain region. Mass spectrometric approaches offer several advantages including higher sensitivity and throughput compared to traditional methods [16-21]. For example, recent neuropeptidomics studies have demonstrated high sensitivity characterization of peptides in diverse tissues such as human brain [22-25], and rat brain [26-30]. Moreover, several protocols for peptide characterization using mass spectrometry as analytical tools have been developed and are used routinely for biomedical applications [16,17,31,32].

Here, we thus sought to characterize the peptide population and its distribution across three important parts of the visual system using tree shrews (Tupaia belangeri) as model animals. Tree shrews are phylogenetically close relatives of primates, and similar to primates, they have a well-developed visual system and therefore are frequently used as animal models for the study of visual structure and function [33]. The three relevant visual centers targeted for neuropeptide characterization in this study are the primary visual cortex (V1), lateral geniculate nucleus (LGN) and superior colliculus (SC); the regions are schematically shown in the Fig. $1 \mathrm{~A}$. We have identified 52 peptides from 21 precursor families in the tree shrew visual system in the three targeted brain regions. This peptide population includes 21 classical neuropeptides that have been previously identified, as well as 26 peptides previously not reported in visual system. We have also used a label free quantification approach to evaluate the relative abundances of a subset of 27 peptides in the studied brain regions. We discuss our findings in relation the possible peptide functions in the visual system in light of previous literature.

\section{Methods}

\subsection{Chemicals}

LC-MS grade acetonitrile and formic acid were purchased from Fisher Scientific (New Jersey, USA) and Fluka (Wisconsin, USA), respectively. Pure water was prepared by GenPure system (TKA, Niederelbert, Germany) Siliconized micro centrifuge tubes (2 mL) were purchased from Eppendorf (Hamburg, Germany). Microcon centrifugal filter devices (Vivacon 500) were purchased from Sartorius AG (Goettingen, Germany Germany).

\subsection{Animals}

Tree shrews (Tupaia belangeri) were used as model animals. They were housed at constant temperature and humidity with free access to food and water. All procedures were in compliance with applicable European Union (EUVD 86/609/ EEC) and Swiss regulations. Three male tree shrews, six years old, were sacrificed by a decapitation after having been anaesthetized with ketamine (Streuli Pharma AG, Uznach, Switzerland).

\subsection{Sample preparation}

During sample preparation, temperature control was used to minimize the degeneration of the endogenous neuropeptides and also to reduce the interference of peptides produced by degradation of proteins. Tree shrew heads were immediately heated up to $80^{\circ} \mathrm{C}$ for $16 \mathrm{~s}$ using microwave irradiation [34]. Their brains were removed and stored at $-80^{\circ} \mathrm{C}$, and visual system parts were dissected from the denatured brain, right and left half brains separately. For each extraction, $10.0 \mathrm{mg}$ of brain tissue was used. Neuropeptides were extracted from 6 samples from both brain hemispheres (V1, LGN and SC). We used a four step extraction procedure that had been developed previously[35]. Briefly, it is based on a distinct gradient of organic phase (methanol) solutions, which were used as follows: firstly an aqueous solution of $0.2 \%$ acetic acid was used two times, then a methanol-water-actic acid solution (20:79.8:0.2, v/v/v) was selected and finally the last extraction part was performed using a methanol-water-acetic acid solution (50:49.8:0:2, v/v/v). Each of the four steps used 15 micro liters of solvent per one mg of brain sample. Before an extraction, samples had been homogenized twice (each time for $20 \mathrm{~s}$ ) within 1 min by a Precellys 24 homogenizer (Bertin Technologies, Montigny-le-Bretonneux, France). After homogenization, they were centrifuged at 22, $000 \mathrm{~g}$ for $60 \mathrm{~min}$ at $4{ }^{\circ} \mathrm{C}$. Before analysis, obtained supernatants from the first two steps (aqueous phase) as well as the supernatants from the last two steps (organic phase) had been mixed together and filtered using a $10 \mathrm{kDa}$ cut-off filter (Vivacon 500, Sartorius AG, Goettingen, Germany) by centrifuging for $90 \mathrm{~min}$ with $14.000 \mathrm{~g}$ at $4{ }^{\circ} \mathrm{C}$. Finally, aqueous and organic peptide extracts were pooled together on a trap column (100 $\mu \mathrm{m}$ ID, $2 \mathrm{~cm}$ long), which had been previously packed with a C18 AQ particles ( $5 \mu \mathrm{m}, 100 \AA$;) ) and afterwards they were injected on an analytical column based on C18 AQ ( $3 \mu \mathrm{m}, 100 \AA)$ stationery phase which was packed in a Picofrit capillary with an emitter tip of $10 \mu \mathrm{m}$ (NewObjective) and analyzed.

\subsection{LC-FT-MS/MS data acquisition}

For all mass spectrometric experiments, LTQ-Orbitrap Discovery (Thermo Fisher Scientific, Bremen, Germany) hyphenated with a 2D NanoLC (Eksigent Technologies, USA) was used. Firstly, samples were injected 5 times on a trap column in a peek column holder (Upchurch). Each injection was $5 \mu \mathrm{l}$ and the interval between injections was $3 \mathrm{~min}$. Trapped analytes were eluted using $2 \%$ acetonitrile and $98 \%$ water containing $0.2 \%$ formic acid. The mobile phase A and B in 


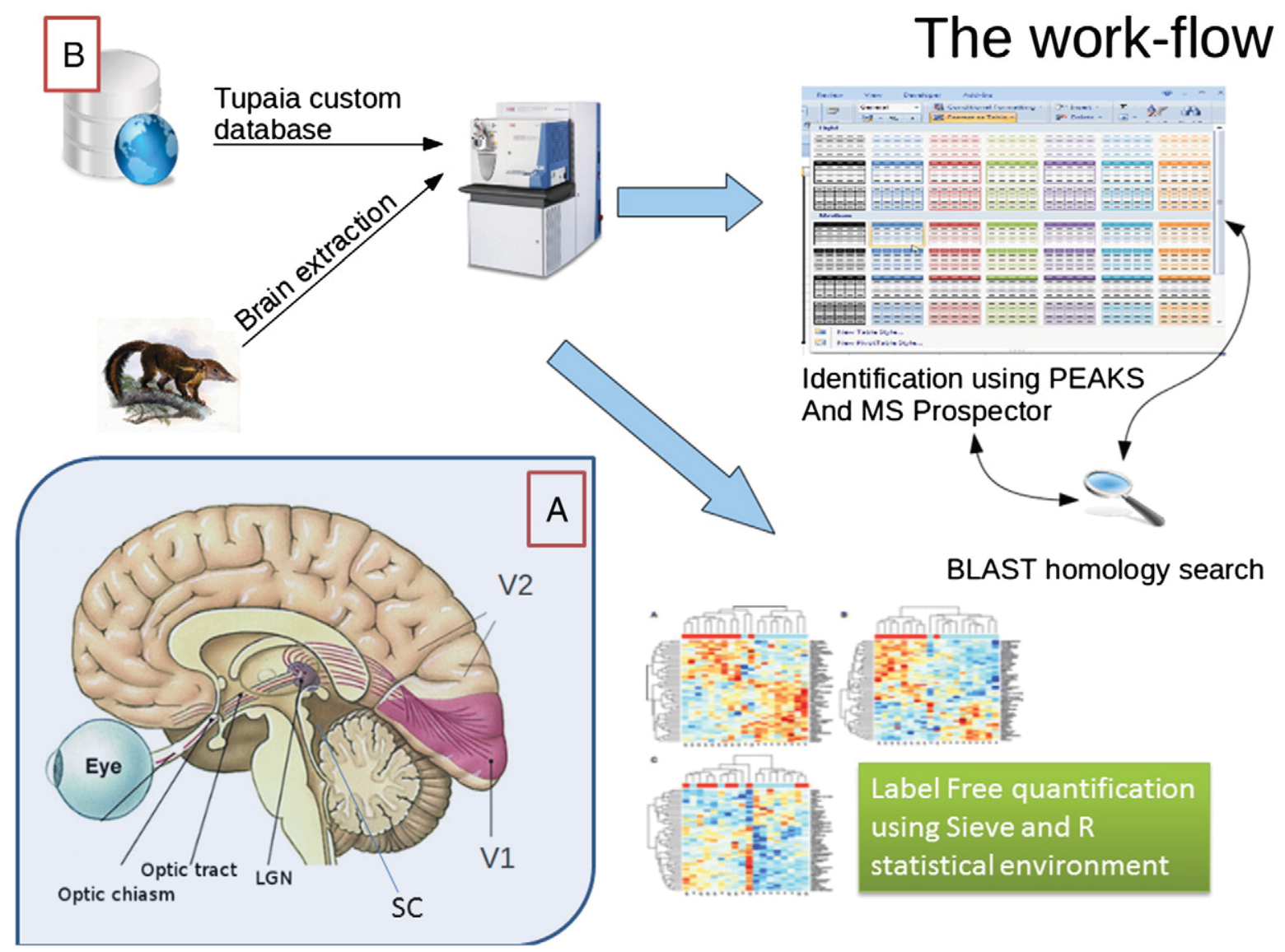

Fig. 1 - A: The scheme of brain sections analyzed in this study. Namely LGN, SC and V1 were investigated. B.: The workflow used in this particular study. Samples were subjected to extraction; extracts were analyzed firstly using DDA mode and afterwards confirmed using Target-mass list mode. Identified entities were subjected to label free quantification.

2D pump were $0.2 \%$ formic acid, and $95 \%$ acetonitrile containing $0.2 \%$ formic acid, respectively. The flow rate was set to $300 \mathrm{nl} / \mathrm{min}$ with the following gradient profile: 0-6 min, $2 \% \mathrm{~B} ; 6-12 \mathrm{~min}, 2-20 \% \mathrm{~B} ; 12-80 \mathrm{~min}, 20-50 \%$ B; $80-85 \mathrm{~min}$, 50-60\% B; 85-90 min, 60-95\% B; and 90-100 min, 95\% B. After each LC-MS run, the LC system was cleaned by an injection of $75 \%$ acetonitrile containing $0.1 \%$ formic acid two times and then conditioned for a next analysis. The mass spectrometric parameters of an LTQ-FTMS instrument consisted of a full FTMS scan event at a mass range from 350 to $2000 \mathrm{~m} / \mathrm{z}$ and data-dependent analysis (DDA) CID MS/MS scans (30,000 resolutions) of the five most abundant peaks from the previous full FTMS scan. All key experimental parameters concerning the mass spectrometric methods were tuned to obtain suitable spectra of selected internal standards (NPY, Neurotensin; concentration level $5 \times 10^{-6} \mathrm{~mol} . \mathrm{L}^{-1}$ ); i.e.: spray voltage, nanospray geometry, heated capillary temperature and ion optics parameters were tuned. Minimum signal threshold of a data dependent scan for the 1st and 5th most intensive ions were set at 5000, 3000, 1000, 1000 and 1000 counts, respectively. Other MS/MS parameters were set as follows: isolation width, $m / z=2$; normalized collision energy, 25\%; activation $\mathrm{Q}, 0.25$; and activation time, $50 \mathrm{~ms}$. Dynamic exclusion was set as a repeat count of 1 , an exclusion duration of $180 \mathrm{~s}$, and a repeat duration of $30 \mathrm{~s}$.
Firstly, all the obtained DDA LC-FTMS/MS data were subjected to Peaks Studio 5.3 (BSI, Canada) hybrid spectral analysis using homemade database of potential neuropeptide precursors of $\mathrm{T}$. belangeri obtained from Euarchontoglires datasets and BLAST homology search using UNIPROT/SWISSPROT databases with false discovery rate control (peptide sequence FDR $<1 \%$ ). Secondly, we used a targeted LC-MS/ MS analysis, with an inclusion list integrated from two independent sources: i) the list of peptides identified in the first step using the DDA approach ii) the previous findings by Petruziziello et al. [36] performed on tree shrew striatum (peptide sequence FDR $<1 \%$ ). The final workflow is shown in the Fig. 1B. Identification of peptides was based on several inclusion criteria: a) the monoisotopic mass of target had to match the theoretical mass in the database with an error no higher than $5 \mathrm{ppm}$. b) the fragmentation spectrum of each target peptide has to be well interpreted and fragment ions has to be measured with an error lower than $0.05 \mathrm{Da}$. c) The isotopic pattern of identified peptides has to match the theoretical model and finally $d$ ) each peptide has to have a clearly defined peak in the respective reconstructed ion chromatogram with $\mathrm{S} / \mathrm{N}$ ratio higher than 3. Finally, each identified peptide was manually checked in the corresponding raw file and only high confidence peptides are reported. Peptide information obtained by a combination of these two approaches is included in the Table 1 . The peptides with $\mathrm{S} / \mathrm{N}$ 
Table 1 - The list of identified peptides present in the visual system of Tupaia belangeri. Identification was performed using the combination of DDA and targeted LC-MS/MS

analysis.

\begin{tabular}{|c|c|c|c|c|c|c|c|c|}
\hline Peptide label & Family & Name & Sequence & $-10 \lg P$ & ppm & $m / z$ & Charge state & Protein accession \\
\hline 15 & ACA polypeptide 1 & & EILNEAYRKVLDQL(-0.98) & 44.23 & 7.7 & 568.33 & 3 & sp|P13589|PACA_RAT \\
\hline 1 & Acyl-CoA-binding protein & & VEKVEELKKKY & 41.31 & 2 & 464.94 & 3 & 15_sp|P11030|ACBP_RAT \\
\hline 17 & CART $^{\mathrm{a}}$ & & Q(-17.03)EDAELQPR.A & 44.85 & 2.6 & 534.75 & 2 & sp|P49192|CART_RAT \\
\hline 2 & Cholecystokinin & & Q(-17.03)SLDPSHRIS & 52.47 & 1.3 & 561.78 & 2 & 18_sp|P09240|CCKN_MOUSE \\
\hline 16 & Chromogranin B & & QYDGVAELDQLLHY & 44.85 & 2.1 & 832.40 & 2 & sp|P16014|SCG1_MOUSE \\
\hline 3 & Delta pre-protachykinin $\mathrm{I}^{\mathrm{a}}$ & & DAGHGQISHKMGYE & 56.46 & 0.2 & 510.57 & 3 & 12_tr|Q9Z0K2|Q9Z0K2_CAVPO \\
\hline 6 & Gamma preprotachykinin & & LPEPFEHLLQ & 41.33 & 2.5 & 611.83 & 2 & sp|P20366|TKN1_HUMAN \\
\hline 4 & Neuroendocrine protein $7 \mathrm{~B} 2^{\mathrm{a}}$ & & SVNPYLQGQRLDNVVA & 48.15 & 2.6 & 886.97 & 2 & 27_tr|Q2PFP9|Q2PFP9_MACFA \\
\hline 20 & Neuropeptide FF receptor 1 & & AMFIAAYALIFLLCMVGNT & 37.78 & 6.5 & 516.03 & 4 & sp|P05408|7B2_HUMAN \\
\hline 42 & Neuropeptide $\mathrm{Y}^{\mathrm{a}}$ & Neuropeptide Y & YPSKPDNPGEDAPAEDMARYYSALRHYINLITRQRY(-.98) & 65.33 & 1.2 & 854.83 & 5 & sp|P07808|NPY_RAT \\
\hline 22 & Neurosecretory-protein VGF & AQEE-30 & AQEEAEAEERRLQEQEELENYIEHVLLRRP & 44.85 & 0.2 & 742.17 & 5 & sp|O15240|VGF_HUMAN \\
\hline 21 & Neurosecretory-protein VGF ${ }^{a}$ & Neuroendocrine regulatory peptide- 2 & EQEELENYIEHVLLRRP & 44.85 & 0.0 & 723.04 & 3 & sp|O15240|VGF_HUMAN \\
\hline & Neurotensin & Neurotensin & Q(-17.03)LYENKPRRPYIL & 43.27 & -0.1 & 558.31 & 3 & 8_sp|Q9D3P9|NEUT_MOUSE \\
\hline & Nucleobindin-2 & & FLEPDSWETLDQQQLF & 49.79 & 3.2 & 499.49 & 4 & sp|Q9JI85|NUCB2_RAT \\
\hline & Prodynorphin $^{a}$ & Dynorphin B & YGGFLRRQFKVVT & 12.5 & -1.5 & 393.48 & 4 & sp|O35417|PDYN_MOUSE \\
\hline & ProEnkephalin A & & GRPEWWMDYQ & 51.56 & 2.1 & 684.30 & 2 & 40_sp|P47969|PENKK_CAVPO \\
\hline & ProEnkephalin $\mathrm{A}^{\mathrm{a}}$ & & VGRPEWWMDY & 56.16 & 0.7 & 669.80 & 2 & 40_sp|P47969|PENK_CAVPO \\
\hline & ProEnkephalin $\mathrm{A}^{\mathrm{a}}$ & Metankephaline & YGGFM & 28.14 & -0.3 & 574.23 & 1 & 40_sp|P47969|PENK_CAVPO \\
\hline & ProEnkephalin $\mathrm{A}^{\mathrm{a}}$ & & YGGFMRF & 51.39 & 1.1 & 439.21 & 2 & 37_sp|P01210|PENK_HUMAN \\
\hline & Proenkephalin-A & & LPSDEEGESYSKEVPEM & 44.85 & 0.4 & 963.42 & 2 & sp|P04094|PENK_RAT \\
\hline & Proenkephalin-A & & ALPSDEEGESYSKEVPEM & 44.85 & 1.7 & 998.94 & 2 & sp|P04094|PENK_RAT \\
\hline & Proenkephalin-A & & LPSDEEGESYSKEVPEME & 44.85 & 1.1 & 1027.94 & 2 & sp|P04094|PENK_RAT \\
\hline & Proenkephalin-A & & ALPSDEEGESYSKEVPEME & 44.85 & 1.8 & 1063.46 & 2 & sp|P04094|PENK_RAT \\
\hline & Proenkephalin-A & & GRPEWWMDYQ & 44.85 & 6.4 & 684.30 & 2 & sp|P04094|PENK_RAT \\
\hline & ProEnkephalin- $\mathrm{A}^{\mathrm{a}}$ & Leu-enkephalin & YGGFL & 22.68 & -1.4 & 556.28 & 1 & 40_sp|P47969|PENK_CAVPO \\
\hline & ProEnkephalin- $A^{a}$ & Met-enkephalin-Arg-Gly-Leu & YGGFMRGL & 59.4 & -0.3 & 450.72 & 2 & 40_sp|P47969|PENK_CAVPO \\
\hline & Pro-melanin-concentrating hormone & & EIGDEENSAKFPI(-.98) & 44.85 & 4.5 & 724.36 & 2 & sp|P14200|MCH_RAT \\
\hline & Pro-melanin-concentrating hormone ${ }^{a}$ & & DFDMLRCMLGRVYRP & 44.85 & 1.5 & 624.64 & 3 & sp|P14200|MCH_RAT \\
\hline & Pro-melanin-concentrating hormone ${ }^{a}$ & Neuropeptide-glutamic acid-isoleucine & EIGDEENSAKFP & 44.85 & 4.5 & 724.36 & 2 & sp|P14200|MCH_RAT \\
\hline & Pro-SAAS & & PPEGVLGALLR & 44.85 & 3.1 & 561.34 & 2 & sp|Q9UHG2|PCSK1_HUMAN \\
\hline & Pro-SAAS & & PPEGVLGALLRV & 44.85 & 4.0 & 610.87 & 2 & sp|Q9UHG2|PCSK1_HUMAN \\
\hline 20 & Pro-SAAS & & PRGEAAGAVQELARALAHLLEAERQE & 44.85 & 3.8 & 697.13 & 4 & sp|Q9UHG2|PCSK1_HUMAN \\
\hline & Pro-SAAS ${ }^{\mathrm{a}}$ & GAV & AVPRGEAAGAVQELARALAHLLEAERQE & 45.1 & 1 & 739.65 & 4 & sp|Q9UHG2|PCSK1_HUMAN \\
\hline (1) & Pro-tachykinin- $1^{\text {a }}$ & Neuropeptide K (frag) & DADSSIEKQVALLKALYGHGQISH & 45.2 & 1.2 & 645.84 & 4 & sp|Q9UHG2|PCSK1_HUMAN \\
\hline 43 & Secretogranin $1^{\mathrm{a}}$ & & Q(-17.03)YDGVAELDQLLHY & 59.2 & -0.7 & 823.89 & 2 & 72_sp|P16014|SCG1_MOUSE \\
\hline 37 & Secretogranin-1 & & QLDLKRQY(-.98) & 37.93 & 6.9 & 531.81 & 2 & sp|P16014|SCG1_MOUSE \\
\hline 24 & Somatostatin & & K.AGCKNFFWKT & 44.85 & 2.7 & 401.20 & 3 & sp|P60042|SMS_RAT \\
\hline 44 & Somatostatin & & APSDPRLRQFLQ & 45.21 & 7.1 & 476.60 & 3 & sp|P60042|SMS_RAT \\
\hline 10 & Somatostatin ${ }^{a}$ & Somatostatin 14 & AGCKNFFWKTFTSC & 55.4 & -0.5 & 820.37 & 2 & 3_tr|Q7TSR3|Q7TSR3_9MURI \\
\hline 39 & Somatostatin ${ }^{a}$ & Somatostatin -28 & SANSNPAMAPRERKAGCKNFFWKTFTSC & 44.85 & 3.2 & 788.13 & 4 & sp|P60042|SMS_RAT \\
\hline 40 & Somatostatin ${ }^{a}$ & Somatostatin - $28(1-12)$ & SANSNPAMAPRER & 44.85 & 3.1 & 622.79 & 2 & sp|P60042|SMS_RAT \\
\hline 12 & Somatostatin ${ }^{a}$ & & FFWKTFTSC & 41.51 & -1.2 & 583.77 & 2 & 3_tr|Q7TSR3|Q7TSR3_9MURI \\
\hline 13 & Substance P & Substance $P$ & RPKPQQFFGLM(-.98) & 42.09 & -3.3 & 674.37 & 2 & 10_sp|Q60541-2|TKN1_MESAU \\
\hline 45 & Tachykinin $1^{\mathrm{a}}$ & Substance P fragment & PKPQQFFGLM(-.98) & 44.85 & 1.4 & 596.32 & 2 & sp|P06767|TKN1_RAT \\
\hline 14 & VIP peptides ${ }^{a}$ & [pGlu16] - VIP (16-28), porcine & Q(-17.03)MAVKKYLNSILN(-.98) & 47.24 & 2 & 752.42 & 2 & 4_sp|P32649|VIP_RABIT \\
\hline
\end{tabular}

$\left({ }^{*}\right)$ Peptide with a substitution of one or two amino acids, identified using homology search.

(-17.03): Glutamination.

(-.98): Amidation.

a a : Error in ppm. 
ratio higher than 10 and CV below 50\% were also relatively quantified.

\subsection{Label free quantification}

For a label free quantification of identified peptides the SIEVE (Version 1.3, Thermo Scientific, U.S.A.) and Matlab 2009b (Mathworks, Natick, Massachusetts, U.S.A.) were used. The label free quantification is based on the direct comparison of peak areas in respective extracted ion chromatograms of previously identified peptides [37]. The data processing includes mainly three steps: i) precursor ion extraction with narrow mass window; ii) peak alignment; and iii) peak area integration. After obtaining the peak areas, further normalization is used for differential analysis (see method below). The Sieve software is a powerful tool for label free quantification and is used for comparison of various compounds levels across diverse samples. In this study, it served as a data alignment tool with following parameters: $\mathrm{m} / \mathrm{z}$ range from 300 to $1500 \mathrm{Da}$, time frame $6 \mathrm{~min}, \mathrm{~m} / \mathrm{z}$ frame $0.02 \mathrm{Da}$ with a peak intensity threshold 10,000.

\subsection{Data normalization}

The process of data normalization presents an important step in the data evaluation. The selection of normalization procedure could dramatically influence the obtained results and thus a careful evaluation of normalization approach has to be accomplished [38]. The normalization across all respective datasets was performed as follows: total abundance of each peptide across all three respective brain areas was calculated (set as 100\%) and the mean expression level was used as a reference point for a calculation of the relative abundance of each peptide in particular brain parts. The label free quantification of respective identified peptides was executed on previously normalized data. All normalizations and calculations were performed using Matlab scripts. Firstly, the $t$-tests were used to compare peptide signal intensities in the left and right half brains and among samples from the same group. We found no statistically significant differences in peptide signal intensities between left and right hemispheres (paired t-tests, $P>0.05$ ).

\section{Results}

\subsection{Wide-ranging identification of neuropeptides in V1,} CSR and LGN

The first aim of the present study is a broad discovery of peptides present in primary visual cortex and two subcortical visual areas, the LGN and SC. In this study, we used an integration of DDA and targeted LC-MS/MS analysis to increase the peptide identification rates. This mode has been previously applied in a proteomics to increase the capability of identification of low abundant peptides. A database search allowed the identification of 52 peptides from 21 pro-hormone families with high confidence. The peptides with their identification details are summarized in Tables 1 and 2. In addition, we found 6 classical neuropeptides that were known to be present in other brain structures but had not been previously reported in the visual system. Classical peptides are labeled with a "\#" symbol in Table 1. Finally, we have identified 7 peptides in the visual system, which exhibit substitutions of one or two amino acid residues in comparison with known peptides in other species. They are labeled with "*" symbol in Table 2. Among our identified peptides, we found 7 peptides with amidation and 5 peptides with glutamination posttranslational modifications (PTMs) (for details see Table 1). PTMs play an important role in the activation of native neuropeptides and also can act as protectors against unwanted degradation processes.

\subsection{Comparative analysis of peptides expressed in differ-} ent visual areas

The second aim of our study was a comparative analysis of the three selected visual areas, based on an evaluation of nor-

\section{Table 2 - The list of peptides identified in the visual system of Tupaia belangeri using homology search.}

\begin{tabular}{|c|c|c|c|c|c|c|c|c|}
\hline Peptide label & Peptide sequence & $\begin{array}{l}\text { Homology } \\
\text { search }\end{array}$ & $\begin{array}{c}\text { SPIDER } \\
\text { score }\end{array}$ & RSD & ppm & $\mathrm{m} / \mathrm{z}$ & $\begin{array}{l}\text { Charge } \\
\text { state }\end{array}$ & Protein accession \\
\hline 46 & SPFDNKLNVEDVDST & a & 43 & 0.07 & 2.3 & 840.4 & 2 & $\begin{array}{l}\text { 49_sp|Q8WXD2| } \\
\text { SCG3_HUMAN }\end{array}$ \\
\hline 47 & SPQLDDEAKELQ & a & 31.5 & 0.17 & 1.7 & 686.8 & 2 & 20_sp|P47969|PENK_CAVPO \\
\hline 48 & SPQLDDEAKEL & a & 28 & 0.18 & -0.1 & 622.8 & 2 & 1_sp|P47969|PENK_CAVPO \\
\hline 49 & SPQLDDEAKE & a & 25.5 & 0.2 & -0.8 & 566.3 & 2 & 1_sp|P47969|PENK_CAVPO \\
\hline 50 & FLGEGYHQVQES & a & 24 & 0.25 & 1.1 & 697.3 & 2 & $\begin{array}{l}\text { 8_tr|B2R5M3| } \\
\text { B2R5M3_HUMAN }\end{array}$ \\
\hline 51 & FLGEGYHQVQE & a & 21 & 0.27 & 0.7 & 653.8 & 2 & $\begin{array}{l}\text { 8_tr|B2R5M3| } \\
\text { B2R5M3_HUMAN }\end{array}$ \\
\hline 52 & K.LDELQKQWKEDLER.Q ${ }^{a}$ & a & 20.77 & 0.29 & 7 & 610.7 & 3 & sp|Q9JI85|NUCB2_RAT \\
\hline $\begin{array}{l}\text { (-17.03): Gluta } \\
\text { (-.98): Amidat } \\
\text { RSD: Relative } \\
\text { ppm: Error in } \\
{ }^{a} \text { Peptide with }\end{array}$ & $\begin{array}{l}\text { nination. } \\
\text { on. } \\
\text { tandard deviance. } \\
\text { pm. } \\
\text { a substitution of one or tw }\end{array}$ & ino acids, & usi & 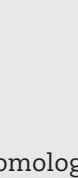 & arch. & & & \\
\hline
\end{tabular}




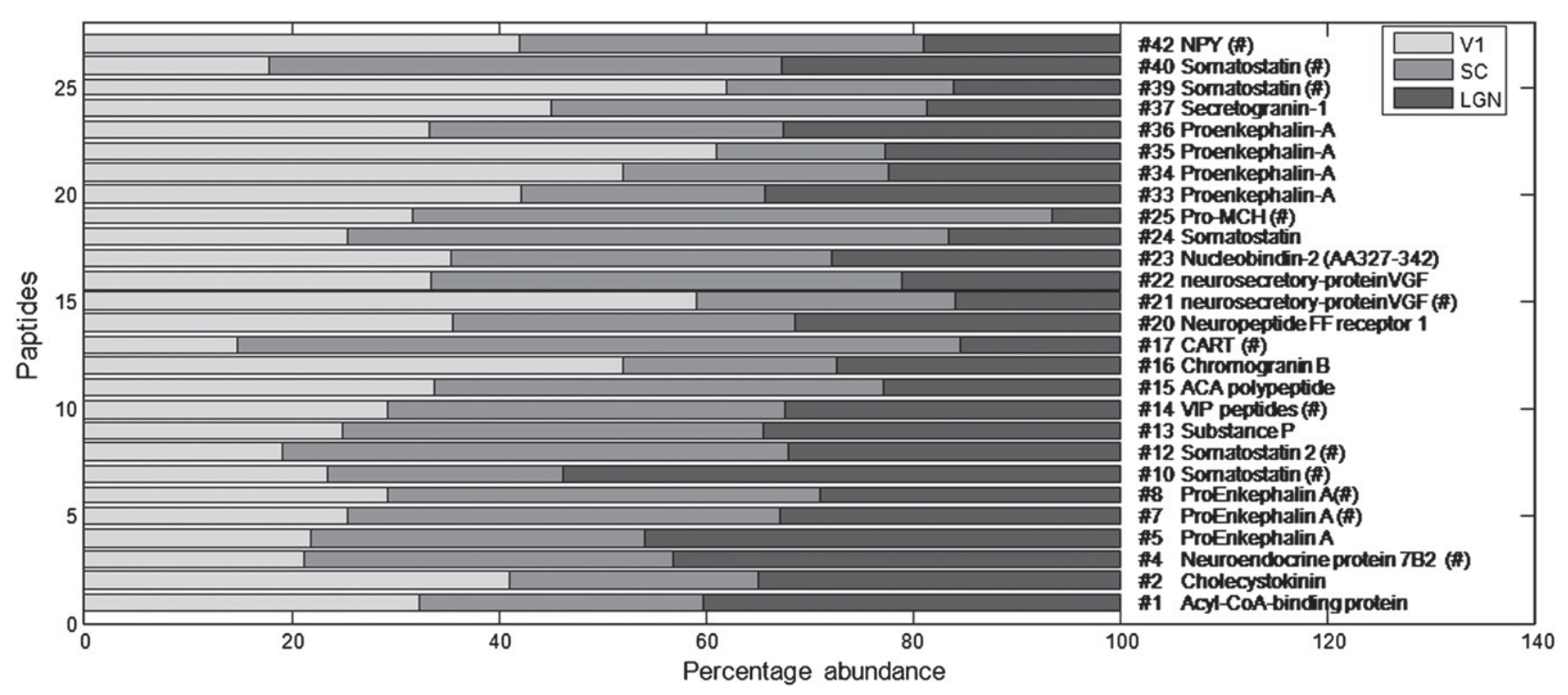

Fig. 2 - Reconstructed histogram with relative expression levels of quantified peptides across target visual centers.

malized peak areas processed according to the protocol described in the Methods section. Fig. 2 summarizes this data by showing label, name and relative abundance for all the peptides in our population, which were subjected to relative quantification ( $\mathrm{S} / \mathrm{N}>10, \mathrm{CV}<50 \%)$. The data are normalized such that the total abundance of each peptide in the three brain regions is expressed as $100 \%$. It is evident that the peptides subjected to quantification are present, albeit at differing concentrations, in all of the targeted brain regions. To examine differences in peptide distribution between areas, we plotted relative peptide abundance across each pair of visual areas: V1 and SC, V1 and LGN and finally, LGN and SC (see Fig. 3). Each dot represents a mean relative expression level (related to a total peak area across studied brain parts and ranging from 0 to $100 \%)$ of the selected peptide, while its label corresponds to the peptide ID in Table 1. We found that both V1 and $\mathrm{SC}$ regions were more rich in peptides compared to the LGN (paired t-tests, $P<0.05$ ), while there was no general difference in peptide abundance between V1 and SC (paired t-test, $P>0.1$ ).

To examine the distribution of targeted peptides, we plotted box-plots for each studied brain region, showing the statistical evaluation of each peptide in the distribution; each box is based on six independent measurements. It is clear that some of the peptides have more specific distribution and are localized mainly in primary visual cortex or one of the subcortical regions. For example Somatostatin 28 (peptide label 39) is more abundant in V1, whereas the rest of this peptide family, including Somatostatin 28 AA1-12 (peptide label 40) and Somatostatin fragment (peptide label 12) are more abundant in subcortical areas, consistent with previous findings using immunoassays [39]. Similarly, Cholecystokinin (peptide label 2) is also observed with higher abundance in V1. On the contrary, we also observed peptides with high abundances in the subcortical areas LGN and SC. An example is the CART peptide (peptide label 17) or Neuroendocrine protein 7B2 (peptide label 4), which are more abundant in SC and LGN, compared to V1. It is worth mentioning that NPY (peptide label 42) is distributed in all studied brain regions and exhibits a higher abundance in V1 compared to SC and V1 (Fig. 4).
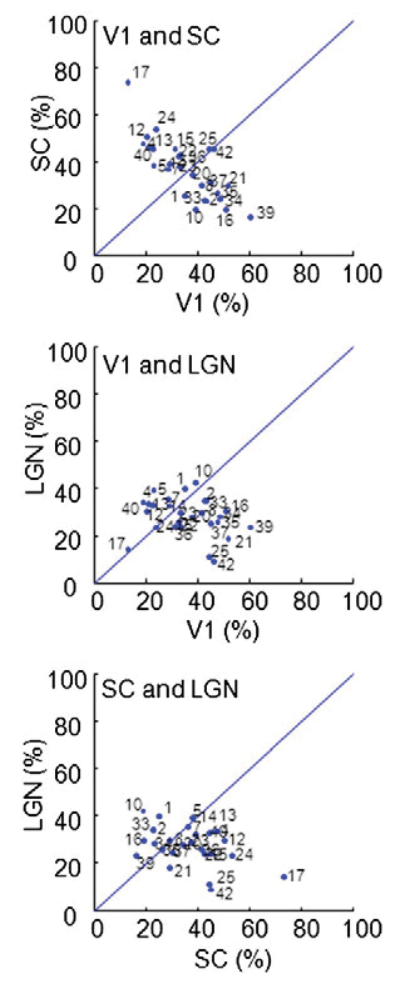

Fig. 3 - Scatter plots of ratios of normalized areas of peptides across each two brain regions. Point labels represent peptide ID found in the Table 1. Primary visual cortex, Superior colliculus and Lateral geniculate nucleus were taken into account. Analysis consists of 27 relatively quantified peptides. 

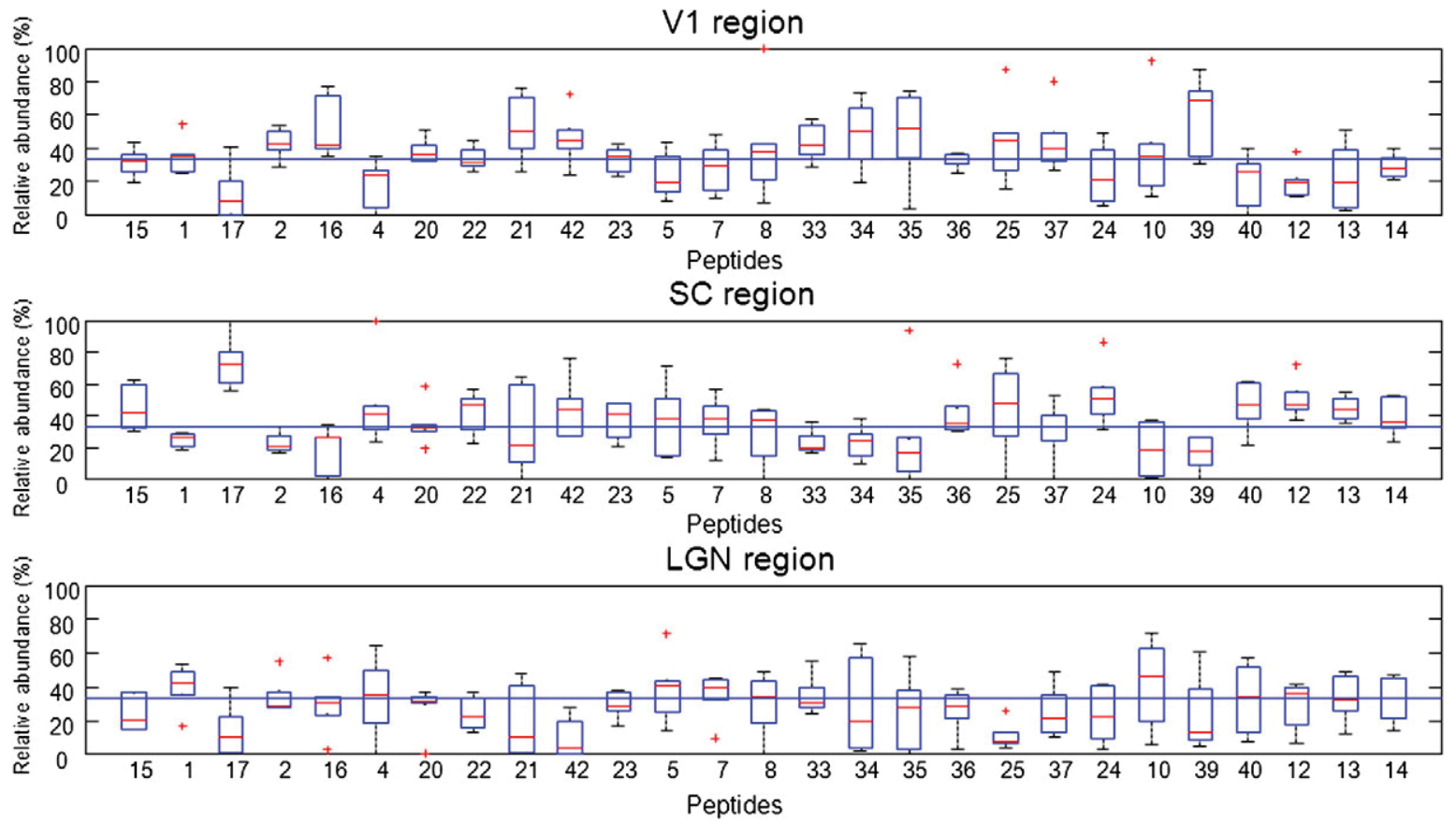

Fig. 4 - Reconstructed bar plots of quantified peptide population. Each brain regions is expressed individually for in-depth comparison. Plus signs stand for outliers; extreme points are represented by the whiskers.

\section{Discussion}

Our study targets the comprehensive characterization of peptide population in the visual system of the tree shrew (T. belangeri). The study of the peptide population in visual system is challenging, mainly due to the low abundance of many peptides compared to peptide-rich brain areas such as the striatum [40]. To provide as complete as possible view of the peptide population in the visual system, we utilized MS-based neuropeptidomics. Our methodological approach utilizes on the integration of data dependent acquisition (DDA) and target-mass list modes, with a mass list based on the previous DDA runs and also on the analysis of peptide rich areas of T. belangeri, i.e. striatum, performed previously [36]. Using this integrated approach, we achieved an identification of 52 peptides from 21 pro-hormone families, including 11 proenkephaline (PENK), 6 Somatostatin (SOM) and three Pro$\mathrm{MCH}$ peptides. A total of 26 peptides, for example GAV, NPK or AQEE-30, were identified in the visual system for the first time. Our differential analysis uncovered that peptides are generally present at lower abundance in the LGN compared to V1 and SC. To our knowledge, this is the first such demonstration in the mammalian visual system, although similar findings have been reported in frogs $[41,42]$. The low abundance of peptides in the LGN might be related to the functional role of this brain area as a relay station of signals from the eye to the brain, where little signal processing occurs in comparison with for example the visual cortex.

We have identified several peptides from the Tachykinin I family [43], including Substance P, Substance P fragment and Neuropeptide K. For example, previous immune-assay studies showed that substance $\mathrm{P}$ is present in SOM positive neurons of the visual cortex during development, while its expression is down-regulated to be absent in adulthood [44]. Our experiment clearly revealed that substance P persists in visual cortex as well as SC and LGN in adult animals, reflecting the superior sensitivity and selectivity of MS-based neuropeptidomics.

We also identified six peptides from the somatostatin family (SOM), including SOM 28, SOM 28 AA1-12 and SOM 14. Somatostatins are expressed by about one third of GABAergic interneurons, and about $90 \%$ of SOM neurons are GABAergic [45]. We observed a high abundance of SOM 28 in the primary visual cortex, consistent with previous findings obtained using radiography evaluation of binding sites in the human brain [46,47], as well as in cat and monkey [48]. In contrast, we found that SOM 28 AA1-12 was present at high abundance in the superior colliculus. This is also consistent with previous findings based on autoradiography measurements in mouse [49], dog [50] and sheep [51] colliculus. This shows that different members of the Somatostatin family are expressed preferentially in different parts of the visual system. Using our MS approach, we are able to simultaneously distinguish six somatostatins, which enhances possibilities for assigning specific physiological functions to particular members of this peptide family.

Neuropeptide Y (NPY) is one of the key classical neuropeptides, playing a key role in the functions of the visual system [52]. NPY levels in visual cortex undergo specific changes during visual system development that are dependent on visual input, and it is thus thought to be important for the processing of visual information [53]. We found that NPY is distributed non-homogenously in the three studied visual system areas, with high abundance observed in V1. Our finding correlates with previous immunochemical results 
in lizard [54] and cat [55], showing a high density of NPY fibers in cortical areas, contrasting low fiber density in other regions such as the thalamus.

Our study also relatively quantified peptides from the ProEnkephaline family, where higher abundances were found in primary visual cortex, compared to SC and LGN. Enkephalines have been previously observed using histochemistry in the visual cortex of cat and turtle [56,57], where a layerdependence has also been described with the highest Enkephalin abundance occurring in layer VI. Although we did not do so here, MS methods can in principle also be used to study peptide distribution across cortical layers. Also consistent with our results are previous reports of Enkephalines in the superior colliculus [58]. While Enkephalins have been mostly associated with addiction and pain, our studies suggest that they may also play an important role in maintaining the function of the visual system. We have also found that Cholecystokinin is more abundant in primary visual cortex, compared to SC and LGN. It has been extensively studied in rat cortex [59], and has been shown to be more abundant in young animals and moderately down-regulated with age. Other peptides such as CART, Pro-MCH or ACA-Peptide also exhibit differential distribution across the studied visual system areas, and more work is needed to describe how they sub serve visual functions during development and adulthood.

We have observed two classical peptides from the VGF family: AQEE-30 and neuroendocrine regulatory peptide-2. They are linked to experience-induced plasticity in the visual cortex and are down-regulated by an inhibition of retinal activity during the critical phase of visual development $[60,61]$. Thus their abundance in adult animals tends to be very low, but as our measurements show these peptides can still be robustly observed in adult animals. Our peptide population also includes a peptide from the vasoactive intestinal polypeptide (VIP) family. VIP is present in many GABAergic neurons, and is thought to be co-released with GABA during interneuron activation. It has been shown that VIP can exert substantial changes in activity of V1 neurons in the cat [62], with effects depending on cortical layer. Thus, in addition to being useful for labeling a specific kind of GABAergic interneuron, VIP can also act as a neurotransmitter in V1 and modify cortical computations. Further work is needed to clarify the role of other peptides on visual processing.

Our study represents the first comprehensive characterization of peptides in the mammalian visual system. Compared to immunochemical or autoradiography methods, our MS-based analysis has demonstrated large coverage and high sensitivity, as evidenced by the detection of a number of peptides whose levels are down-regulated in the adult animals that we studied. In addition, MS-based methods can distinguish even closely related peptides, thus offering superior selectivity and the possibility to ascribe physiological functions to particular members of a peptide family with high confidence.

\section{Concluding remarks}

The presented study deals with the investigation of expression levels of peptides in the tree shrew visual system areas
V1, LGN and SC. Using a synergy of DDA (data dependent analysis) and targeted nanoLC-MS/MS approaches, we have identified a total 53 peptides from 21 distinctive pro-hormone families. We have discovered that a considerable fraction of our peptides exhibits differential abundance across the three visual system areas we investigated. The LGN was found to be the least rich in peptides, whereas generally similar peptide abundance was observed in SC and V1. Our study uncovered differential expression levels of some key peptides across targeted regions. Our results demonstrated that in the mammalian brain, NPY, SOM 28 and some of the opioid peptides, were more abundant in V1, while SOM 28 AA1-12 and a peptide from the Acyl-CoA-binding protein family were more abundant in the superior colliculus. We have shown that our approach can be used for the differential analysis of a population of peptides in the visual system, which now opens the possibility to systematic investigations of how behavioral or pharmacological manipulations impact visual system peptide levels.

\section{Acknowledgements}

This work is supported by the Research Project of the Ministry of Education of the Czech Republic No. MSM6198959216 and by the SNF R'Equip 316000-121308 and a EURYI award to GR.

\section{R E F E R E N C E S}

[1] Villegas R, Castillo C, Villegas GM. The origin of the neuron: the first neuron in the phylogenetic tree of life. Astrobiology: Origins from the Big-Bang to Civilisation 2000:195-211.

[2] Vanderhaeghen JJ, Lotstra F, Schiffmann SN, Mailleux P, Przedborski S, Galas MC, et al. Visualization of Neuropeptides and Neurotransmitters in the Human Brain - Applications to Development and Pathology. Wenner-Gr C, 53; 1989. p. 31-40.

[3] Strand FL. Neuropeptides: regulators of physiological processes. MIT Press: Cambridge, Mass; 1999.

[4] Schulkin J. The neuroendocrine regulation of behavior. Cambridge, UK ; New York, NY, USA: Cambridge University Press; 1999.

[5] Hoyle CHV. Neuropeptides : essential data. Chichester; New York: Wiley; 1996.

[6] Ellsworth SJ, Schuster RC. Appetite and nutritional assessment. Hauppauge, NY: Nova Science; 2009.

[7] Lajtha A. Handbook of neurochemistry and molecular neurobiology. Amino acids and peptides in the nervous system. 3rd ed. New York: Springer; 2007.

[8] Real MA, Heredia R, Labrador Mdel C, Davila JC, Guirado S. Expression of somatostatin and neuropeptide $\mathrm{Y}$ in the embryonic, postnatal, and adult mouse amygdalar complex. J Comp Neurol 2009;513:335-48.

[9] Lim R, Lajtha A. Handbook of neurochemistry and molecular neurobiology. Neuroactive proteins and peptides. 3rd ed. New York, NY: Springer; 2006.

[10] Hensch TK, Fagiolini M. Excitatory-inhibitory balance and critical period plasticity in developing visual cortex. Prog Brain Res 2005;147:115-24.

[11] Chen JL, Lin WC, Cha JW, So PT, Kubota Y, Nedivi E. Structural basis for the role of inhibition in facilitating adult brain plasticity. Nat Neurosci 2011;14:587-94. 
[12] Tanaka M. Newly-discovered neuropeptides in the hypothalamus. FEBS J 2010;277:4989.

[13] Elalouf JM, Bernay B, Gaillard MC, Guryca V, Emadali A, Kuhn $\mathrm{L}$, et al. Discovering new bioactive neuropeptides in the striatum secretome using in vivo microdialysis and versatile proteomics. Mol Cell Proteomics 2009;8:946-58.

[14] Fink G, Harmar AJ. Neuropeptides : a methodology. Chichester England; New York: Wiley; 1989.

[15] Rutishauser J. Copeptin: diagnostic parameter, biomarker, or both? Therapeutische Umschau Revue therapeutique 2009;66:731-4.

[16] Merighi A. Neuropeptides: methods and protocols. New York: Springer; 2011.

[17] Soloviev M. Peptidomics : methods and protocols. New York, NY: Humana Press; 2010.

[18] Lipton MS, Paša-Tolić L. Mass spectrometry of proteins and peptides: methods and protocols. 2nd ed. New York: Humana; 2009.

[19] Romanova EV, Lee JE, Kelleher NL, Sweedler JV, Gulley JM. Mass spectrometry screening reveals peptides modulated differentially in the medial prefrontal cortex of rats with disparate initial sensitivity to cocaine. AAPS J 2010;12:443-54.

[20] Sweedler JV, Li L, Rubakhin SS, Alexeeva V, Dembrow NC, Dowling $\mathrm{O}$, et al. Identification and characterization of the feeding circuit-activating peptides, a novel neuropeptide family of aplysia. J Neurosci 2002;22:7797-808.

[21] Falth M, Skold K, Svensson M, Nilsson A, Fenyo D, Andren PE. Neuropeptidomics strategies for specific and sensitive identification of endogenous peptides. Mol Cell Proteomics 2007;6:1188-97.

[22] Meyer-Lindenberg A, Domes G, Kirsch P, Heinrichs M. Oxytocin and vasopressin in the human brain: social neuropeptides for translational medicine. Nat Rev Neurosci 2011;12:524-38.

[23] Meyer-Lindenberg A. Impact of prosocial neuropeptides on human brain function. Advances in vasopressin and oxytocin: from genes to behaviour to disease, 170; 2008. p. $463-70$.

[24] Frankel PS, Alburges ME, Bush L, Hanson GR, Kish SJ. Brain levels of neuropeptides in human chronic methamphetamine users. Neuropharmacology 2007;53:447-54.

[25] Takahashi K, Satoh F, Totsune K, Sone M, Mizuno Y, Ohneda $\mathrm{M}$, et al. Neuropeptides in the human brain. Funct Neurol 1996;11:65-98.

[26] Mokrushin AA, Tokarev AV. Endogenous neuropeptides are involved in long-term potentiation and depression in rat-brain olfactory cortex slices. J Neurochem 1995;65:S120-S.

[27] Theodorsson E, Mathe AA, Stenfors C. Brain neuropeptides tachykinins, neuropeptide-Y, neurotensin and vasoactive intestinal polypeptide in the rat-brain - modifications by Ect and indomethacin. Prog Neuropsychopharmacol Biol Psychiatry 1990;14:387-407.

[28] Zhang X, Liu Y, Feng C, Yang S, Wang Y, Wu AS, et al. Proteomic profiling of the insoluble fractions in the rat hippocampus post-propofol anesthesia. Neurosci Lett 2009;465:165-70.

[29] Skold K, Svensson M, Kaplan A, Bjorkesten L, Astrom J, Andren PE. A neuroproteomic approach to targeting neuropeptides in the brain. Proteomics 2002;2:447-54.

[30] Rossbach U, Nilsson A, Falth M, Kultima K, Zhou Q Hallberg $\mathrm{M}$, et al. A quantitative peptidomic analysis of peptides related to the endogenous opioid and tachykinin systems in nucleus accumbens of rats following naloxoneprecipitated morphine withdrawal. J Proteome Res 2009;8: 1091-8.

[31] Levine BA. Neuropeptide research trends. New York: Nova Biomedical Books; 2007.

[32] Farley EP. Progress in neuropeptide research. New York: Nova Biomedical Books; 2007.

[33] Tigges J, Shantha TR. A stereotaxic brain atlas of the tree shrew (Tupaia glis). Baltimore: Williams \& Wilkins Co.; 1969.
[34] Che FY, Lim J, Pan H, Biswas R, Fricker LD. Quantitative neuropeptidomics of microwave-irradiated mouse brain and pituitary. Mol Cell Proteomics 2005;4:1391-405.

[35] Zhang X, Petruzziello F, Zani F, Fouillen L, Andren PE, Solinas G, et al. High identification rates of endogenous neuropeptides from mouse brain. J Proteome Res 2012, doi:10.1021/pr3001699.

[36] Petruzziello F, Fouillen L, Wadensten H, Kretz R, Andren PE, Rainer G, et al. Extensive characterization of Tupaia belangeri neuropeptidome using an integrated mass spectrometric approach. J Proteome Res 2012;11:886-96.

[37] Wang GH, Wu WW, Zeng WH, Chou CL, Shen RF. Label-free protein quantification using LC-coupled ion trap or FT mass spectrometry: reproducibility, linearity, and application with complex proteomes. J Proteome Res 2006;5:1214-23.

[38] Kultima K, Nilsson A, Scholz B, Rossbach UL, Falth M, Andren PE. Development and evaluation of normalization methods for label-free relative quantification of endogenous peptides. Mol Cell Proteomics 2009;8:2285-95.

[39] Fung SJ, Webster MJ, Sivagnanasundaram S, Duncan C, Elashoff M, Weickert CS. Expression of interneuron markers in the dorsolateral prefrontal cortex of the developing human and in schizophrenia. Am J Psychiatry 2010;167:1479-88.

[40] Che FY, Lim J, Pan H, Biswas R, Fricker LD. Quantitative neuropeptidomics of microwave-irradiated mouse brain and pituitary. Mol Cell Proteomics 2005;4:1391-405.

[41] Lazar G. Peptides in frog brain areas processing visual information. Microsc Res Tech 2001;54:201-19.

[42] Debski EA. Distribution and regulation of substance P-related peptide in the frog visual system. Microsc Res Tech 2001;54: 220-8.

[43] Khawaja AM, Rogers DF. Tachykinins: receptor to effector. Int J Biochem Cell Biol 1996;28:721-38.

[44] Mousa SA, Shaqura M, Schaper J, Treskatsch S, Habazettl H, Schafer M, et al. Developmental expression of delta-opioid receptors during maturation of the parasympathetic, sympathetic, and sensory innervations of the neonatal heart: early targets for opioid regulation of autonomic control. J Comp Neurol 2011;519:957-71.

[45] Gonchar Y, Burkhalter A. Three distinct families of GABAergic neurons in rat visual cortex. Cereb Cortex 1997;7: 347-58.

[46] Thoss VS, Piwko C, Probst A, Hoyer D. Autoradiographic analysis of somatostatin SRIF(1) and SRIF(2) receptors in the human brain and pituitary. Naunyn-Schmiedebergs Arch Pharmacol 1997;355:168-76.

[47] Thoss VS, Piwko C, Hoyer D. Somatostatin receptors in the Rhesus monkey brain: localization and pharmacological characterization. Naunyn-Schmiedebergs Arch Pharmacol 1996;353:648-60

[48] Rosier AM, Leroux P, Vaudry H, Orban GA, Vandesande F. Distribution of somatostatin receptors in the cat and monkey visual-cortex demonstrated by invitro receptor autoradiography. J Comp Neurol 1991;310:189-99.

[49] Ma WP, Liu BH, Li YT, Huang ZJ, Zhang LI, Tao HW. Visual representations by cortical somatostatin inhibitory neuronsselective but with weak and delayed responses. J Neurosci 2010;30:14371-9

[50] Pego-Reigosa R, Covenas R, Tramu G, Pesini P. Distribution of somatostatin-28 (1-12) immunoreactivity in the diencephalon and the brainstem of the dog. Anat Embryol (Berl) 2001;203: 61-76.

[51] Fodor M, Slama A, Guillaume V, Videau C, Csaba Z, Oliver C, et al. Distribution and pharmacological characterization of somatostatin receptor binding sites in the sheep brain. J Chem Neuroanat 1997;12:175-82.

[52] McDonald JK, Parnavelas JG, Davies SW, Cavanagh ME. Measurements of somatostatin and neuropeptide $Y$ in the visual cortex of monocularly deprived rats. Exp Neurol 1993;123:216-21. 
[53] Antonopoulos J, Papadopoulos GC, Michaloudi H, Cavanagh ME, Parnavelas JG. Postnatal development of neuropeptide Y-containing neurons in the visual cortex of normal- and dark-reared rats. Neurosci Lett 1992;145:75-8.

[54] Medina L, Marti E, Artero C, Fasolo A, Puelles L. Distribution of neuropeptide Y-like immunoreactivity in the brain of the lizard Gallotia galloti. J Comp Neurol 1992;319:387-405.

[55] Borostyankoi ZA, Gorcs TJ, Hamori J. Immunocytochemical mapping of NPY and VIP neuronal elements in the cat subcortical visual nuclei, with special reference to the pretectum and accessory optic system. Anat Embryol 1999;200:495-508.

[56] Bakalkin GY, Pivovarov AS, Kobylyansky AG, Nesterenko PN, Yarygin KN. Lateralization of opioid receptors in turtle visual-cortex. Brain Res 1989;480:268-76.

[57] Gu Q Cynader MS. Immunocytochemical localization of enkephalin in the cat visual-cortex. Brain Res 1993;620:155-8.
[58] Mensah-Brown EPK, Garey LJ. The superior colliculus of the camel: a neuronal-specific nuclear protein (NeuN) and neuropeptide study. J Anat 2006;208:239-50.

[59] Demeulemeester H, Vandesande F, Orban GA, Brandon C, Vanderhaeghen JJ. Heterogeneity of GABAergic cells in cat visual cortex. J Neurosci 1988;8:988-1000.

[60] Black IB, Alder J, Thakker-Varia S, Bangasser DA, Kuroiwa M, Plummer MR, et al. Brain-derived neurotrophic factorinduced gene expression reveals novel actions of VGF in hippocampal synaptic plasticity. J Neurosci 2003;23:10800-8.

[61] Alder J, Thakker-Varia S. Neuropeptides in depression: role of VGF. Behav Brain Res 2009;197:262-78.

[62] Murphy PC, Grieve KL, Sillito AM. Effects of vasoactive intestinal polypeptide on the response properties of cells in area 17 of the cat visual cortex. J Neurophysiol 1993;69:1465-74. 